\title{
Fusion and Fission in the Visual Pathways
}

\author{
P. KAPOSVÁRI ${ }^{{ }^{*}}$, A. BOGNÁR ${ }^{{ }^{*}}$, P. CSIBRI $^{\mathbf{1}}$, G. UTASSY $^{\mathbf{1}}$, GY. SÁRY $^{\mathbf{1}}$ \\ ${ }^{*}$ These authors contributed equally to this study \\ ${ }^{1}$ Department of Physiology, Faculty of Medicine, University of Szeged, Szeged, Hungary
}

Received October 30, 2013

Accepted March 21, 2014

On-line June 5, 2014

\begin{abstract}
Summary
Inconsistent information from different modalities can be delusive for perception. This phenomenon can be observed with simultaneously presented inconsistent numbers of brief flashes and short tones. The conflict of bimodal information is reflected in double flash or fission, and flash fusion illusions, respectively. The temporal resolution of the vision system plays a fundamental role in the development of these illusions. As the parallel, dorsal and ventral pathways have different temporal resolution we presume that these pathways play different roles in the illusions. We used pathway-optimized stimuli to induce the illusions on separately driven visual streams. Our results show that both pathways support the double flash illusion, while the presence of the fusion illusion depends on the activated pathway. The dorsal pathway, which has better temporal resolution, does not support fusion, while the ventral pathway which has worse temporal resolution shows fusion strongly.
\end{abstract}

\section{Key words}

Audio-visual integration • Double flash • Fusion • Illusion

\section{Corresponding author}

P. Kaposvári, Department of Physiology, Faculty of Medicine, University of Szeged, H-6720, Szeged, Dóm tér 10, Hungary. Fax: +36 62545 842. E-mail: kpsvri@gmail.com

\section{Introduction}

Visual stimuli, presented simultaneously, can interfere with each other even if they are positioned far away from the attended stimulus. Effects on the perception of the attended stimulus can also be demonstrated if the two stimuli belong to different modalities, e.g., visual and auditory (Wilson 1987), or even visual and haptic (Ernst et al. 2000, Wozny et al. 2008). The combination of one or two brief flashes simultaneously presented with one or two short tones results in two inconsistent conditions. The first is where one flash is presented with two tones; in this case, the second tone added induces an illusion of a second flash (Shams et al. 2000). The second is where two flashes are presented with one tone; in this case, the tone can induce the perception of two flashes fusing into one (Andersen et al. 2004, Watkins et al. 2007). Several studies demonstrated cortical and subcortical activities behind the behavioral observation. Electrophysiological evidence shows that the illusion induced extra activity can be detected over the primary visual cortex (Watkins et al. 2006, 2007). Magnetoencephalography (MEG) experiments, for example, have shown that the activity of cortical visual areas can be modulated with sound stimuli at occipital, parietal and anterior regions (Shams et al. 2005). Electroencephalography (EEG) studies have found that, during the illusion, oscillatory and induced gamma band responses were significantly higher, and audiovisual interactions were supra-additive (Bhattacharya et al. 2002). EEG and evoked potential experiments have shown that, during the illusory flash, perceptual activity was modulated strongly and with short latency in trials where the illusory flash was perceived (Shams et al. 2001). Also, it has been found that the potentials observed after the illusory flash were similar to those observed after real flashes. This indicates that the underlying neuronal mechanism is similar in both cases and is a result of a very rapid interaction between auditory and visual areas initiated by the second sound (Mishra et al. 2007, 2008). FMRI data have shown illusory flash related brain activity in superior colliculus, 
the primary visual cortex, and in the right superior temporal sulcus (STS, Watkins et al. 2006, 2007). Also, another group found fusion illusion related activity in superior temporal cortex (Mishra et al. 2008). These studies suggest that such processing of bimodal information could be based on communication between the primary visual cortex, superior temporal sulcus (STS) and primary auditory cortex (Mishra et al. 2008, Watkins et al. 2006, 2007). Since these areas serve as a target for the cortical visual streams as well, it would be interesting to know how the two visual pathways contribute to the information exchange between the primary visual cortex and, for instance, the STS.

The interaction-related activity of the superior colliculus (Watkins et al. 2006) shows the M-pathway is involved in audio-visual interaction. This is in accordance with observations suggesting that the enhanced visual detection can be attributed to the magnocellular system, as proposed by former and recent studies (Jaekl and SotoFaraco 2010, Meredith 2002). Whether the P pathway or ventral stream contributes to the double flash and fusion illusions is unknown.

We do not know to what extent the different pathways are involved in the two illusions or how the interaction spreads between the two pathways during these illusions.

The M pathway is known for processing achromatic, low contrast stimuli very fast (Bullier and Nowak 1995, Maunsell et al. 1990, Merigan and Maunsell 1993, Shapley 1990).

The M-pathway can be selectively stimulated with stimuli having low spatial frequency and low contrast; however, these weak stimuli cannot drive this pathway at full extent (Derrington and Lennie 1984, Kaplan and Shapley 1986, Lee et al. 1995, Leonards and Singer 1997). According to a recent theory the $M$ pathway can send information into the inferotemporal cortex through the orbitofrontal areas, thus preparing it for the incoming, slower activation through the P pathway (Kveraga et al. 2007).

In contrast, the $\mathrm{P}$ pathway conducts information about colors and high spatial frequencies with a much slower speed and needs much higher contrast (about $8 \%$ at least) when detecting achromatic stimuli (Hicks et al. 1983, Tootell et al. 1988). The parvocellular pathway has worse temporal resolution (Derrington and Lennie 1984) as compared to the M pathway. (The magnocellular units in the macaque lateral geniculate body have the highest sensitivity for stimuli modulated at temporal frequencies close to $20 \mathrm{~Hz}$, while the optimum for parvocellular units is close to $10 \mathrm{~Hz}$.) Stimuli containing high spatial frequencies can drive this system selectively. Since the $\mathrm{P}$ pathway is responsible for coding color information, it can also be selectively stimulated with isoluminant color stimuli (Tobimatsu et al. 1996).

In this study, we investigated how the magnoand parvocellular pathways contribute to the development of the double flash and flash fusion illusions. Making a distinction between two consecutively presented flashes depends on the temporal resolution capacities of the observer. Indeed, Metha and Mullen (1996) showed higher performance of the flicker detection in achromatic condition compared to the condition with red-green stimuli. The auditory information can be more effective on a slower, less sensitive system. Therefore, the two visual pathways with different temporal resolutions could be involved with different degrees in the two illusions; in other words, STS could receive information through different pathways depending on the type of integration.

We used pathway-specific visual stimuli simultaneously with pure, meaningless tones as input for the integration processes. We hypothesized that the parallel pathways in accordance to their temporal resolution play different roles in the illusions. Multimodal stimuli - especially in temporal context - are frequently used to get better understanding of how different modalities can combine and influence the processing of each other. The double flash and fusion illusions are appropriate phenomenons to investigate the temporal aspect of audio-visual integration. Still, it is not clear which mechanisms of the visual machinery contribute to these findings. The next logical step in understanding the neuronal background of the illusory flash phenomenon could be an approach where we make a functional distinction between the cortical pathways. We are aware of the fact that this distinction (especially at higher levels than the primary visual cortex) is less and less valid, but this might serve as a good working frame for collecting more data about the double flash and flash fusion and the underlying mechanisms.

\section{Methods}

\section{Participants}

Thirty-four healthy naive volunteers participated in the study. They had normal or corrected vision and normal hearing, with no known neurological disorders. Their color vision was found to be good by the Ishihara 
color perception test. Each one signed an informed consent before the test. The experiment fulfilled the requirements of the Ethical Committee for Experimental Procedures of the University of Szeged.

Seventeen (12 females; mean age: 22.6 years) of thirty-four subjects participated in the test with central visual stimulation, and the other seventeen subjects (13 females; mean age: 22.2 years) with peripheral visual stimulation.

\section{Stimuli and procedure}

Subjects were seated in a sound-attenuated dark room. Their heads were rested on a chin and forehead support. The eyes of the subjects were $57 \mathrm{~cm}$ away from the computer screen and the speakers.

The stimuli were presented on a CRT monitor (ViewSonic PF815). The diameter and the resolution of the screen were 21 inches and 800 x 600 at $60 \mathrm{~Hz}$, respectively.

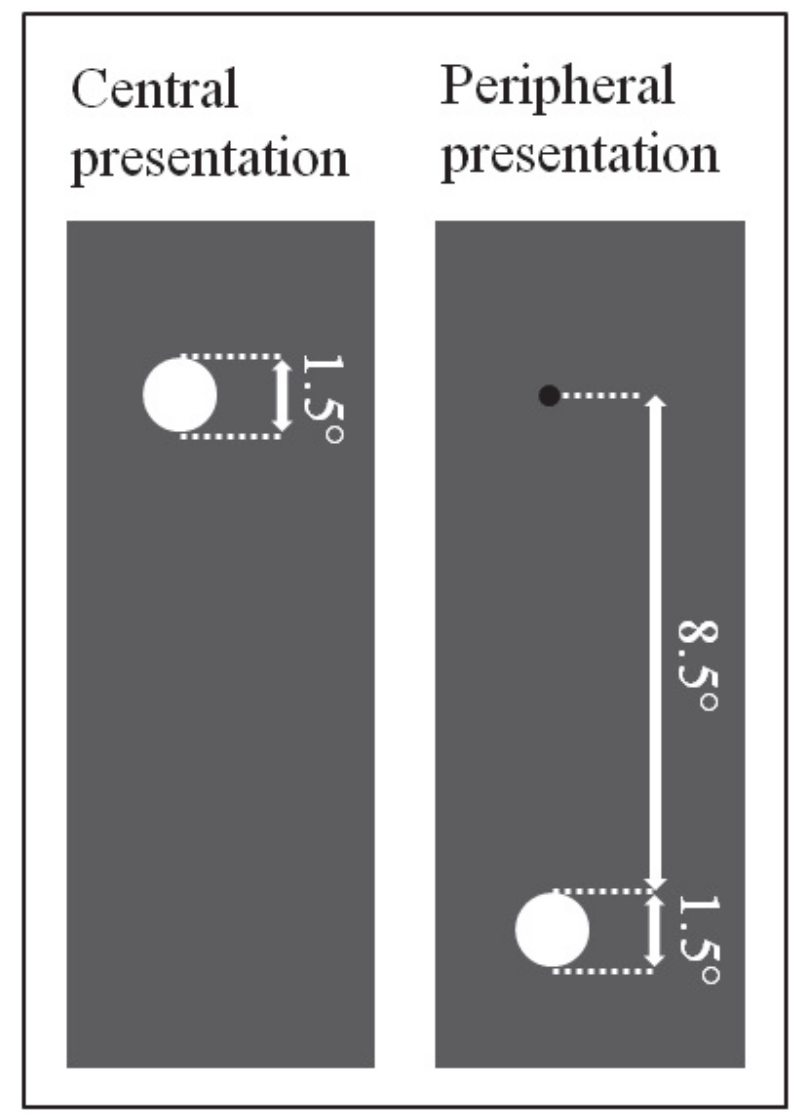

Fig. 1. Location and luminance of the stimuli. Grey scaled versions of the presented stimuli in central and peripheral conditions. In both positions the visual angle of the presented disc was $1.5^{\circ}$ on a green background. In the high contrast conditions the contrast was $75 \%$. In the low contrast conditions the contrast was $9 \%$. In the isoluminant conditions a red disc was presented on the background. The little dark point on the upper part of the panel represents the fixation point in the peripheral condition.
The two computer speakers were positioned on both sides of the monitor, symmetrically, at $25^{\circ}$ from the fixation point. Subjects had to fix their gaze at the middle of the monitor, thus the size and position of the visual stimuli were held constant on the retina. A disc subtending a visual angle of $1.5^{\circ}$ was displayed in a central or peripheral position as visual stimulus for the two groups of the subjects (central and peripheral stimulation, respectively).

All stimuli were presented on a uniform green background $\left(8.9 \mathrm{~cd} / \mathrm{m}^{2}\right)$. We used four conditions with high contrast $(\mathrm{HC})$ with white disc $\left(63 \mathrm{~cd} / \mathrm{m}^{2}\right.$, contrast $75 \%$ ), low contrast (LC) with grey disc $\left(9.7 \mathrm{~cd} / \mathrm{m}^{2}\right.$, contrast 9\%), subjective isoluminant (S-iso) and physically isoluminant (P-iso) with red disc in both positions (Fig. 1). In the above mentioned experiments the same size of stimuli were used with high contrast. So we created a high contrast condition to make our results comparable with earlier findings. With low contrast stimuli we can drive the $M$ pathway. We chose a relatively high contrast value to exclude the big variability between subjects in the control condition. The contrast values were calculated using the Michelson equation.

We used two types of isoluminant conditions. Both of them had color information, thus they drove the $\mathrm{P}$ pathway. The physically isoluminant stimuli have only color information, but the different colors drive the visual system with different strength. The subjective isoluminant stimulus is known as it can drive most selectively the $\mathrm{P}$ pathway (Skottun 2013). In the peripheral task a fixation point was placed in the middle of the screen and the stimulus disc was presented it at $9.25^{\circ}$ eccentricity (Watkins et al. 2006). In the central task, the disc was presented in the middle of the screen without fixation point.

To measure the subjective isoluminance level of the red disc compared to the green background we used the method of heterochromatic flicker photometry (HFP). Red and green discs were reversed at $14 \mathrm{~Hz}$ (Kveraga et al. 2007) on a gray background. The size and position of the disc was the same as we used for the main experiment. We created a range of red intensities and presented them one by one to the participants during the HFP test. Since isoluminance changes across the retina (Bilodeau and Faubert 1997), the test was performed both in the central and the peripheral retina location as well. The luminance value of the green was the same as the background we used in the main experiment. The subjects 
viewed the display binocularly and were asked to choose the intensity value of red where minimal or no flicker was perceived. The isoluminant point was the average of at least three consecutive, independent and consequent measurements.

The central and peripheral tasks contained four blocks (four main conditions, HC, LC, S-iso, P-iso), and followed each other randomly to reduce the chance of fatigue or learning. One block contained 6 subconditions: 6 variations of flashes and tones (one flash, one flash with one tone, one flash with two tones, two flashes, two flashes with one tone, and two flashes with two tones). One subcondition consisted of 40 repetitions of trials, thus one block contained 240 semirandom-presented trials.

The presentation of the trial started with the green background. On this background, after $200 \mathrm{~ms}$ one or two discs were presented for 1 frame $(17 \mathrm{~ms})$ with one or two tones, according to the given condition. The stimulus onset asynchrony (SOA) between two flashes was $85 \mathrm{~ms}$. The duration of the tones $(3.5 \mathrm{kHz}, 70 \mathrm{~dB}$ SPL) was $10 \mathrm{~ms}$, and the first one was presented at the same time as the first flash. The SOA between the two tones was $85 \mathrm{~ms}$. The previously mentioned experiments used auditory and visual stimuli slightly shifted in time but as reported the two designs with simultaneously presented or shifted stimuli resulted only in slight differences (Watkins et al. 2007).

After the presentation of flashes and tones the subject was asked to decide whether one or two discs were displayed independently of the tones and press the left (one flash) or right arrow (two flashes) button on the keyboard with the dominant hand. After the subject pressed a button, an isoluminant grey background $\left(8.9 \mathrm{~cd} / \mathrm{m}^{2}\right)$ appeared as intertrial interval for $1000 \mathrm{~ms}$ (Fig. 2). Feedback was not provided about the correctness of the response.

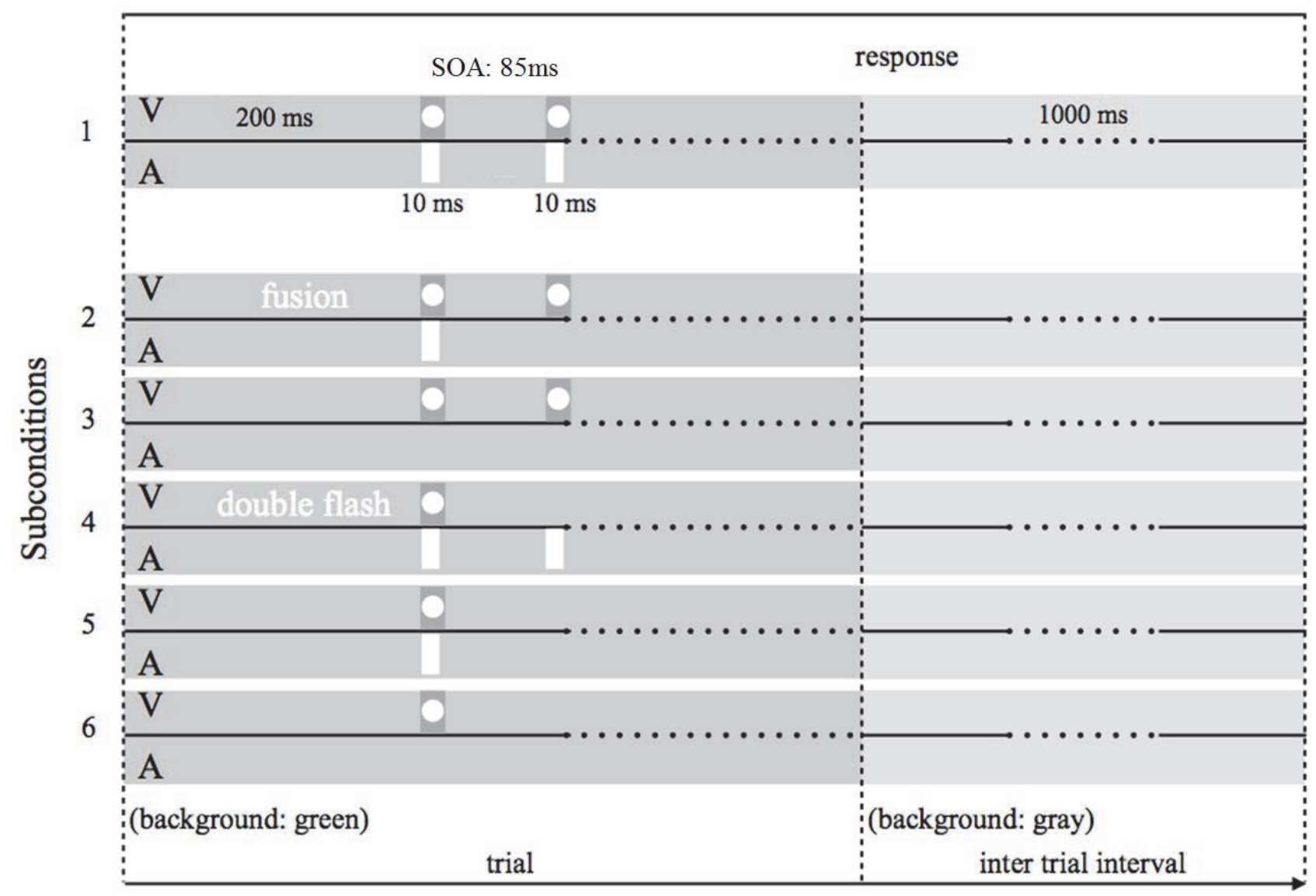

Fig. 2. Design of the task. Stimuli were presented were on green background according to the given subconditions. 1: two discs were presented with two tones; 2: two discs were presented with one tone; 3: two discs were presented without any tones; 4: one disc was presented with two tones; 5: one disc was presented with one tone; 6 : one disc was presented without any tones. The duration of the tone was $10 \mathrm{~ms}$ and the SOA for the two tones was $85 \mathrm{~ms}$. The duration of the visual stimuli was $17 \mathrm{~ms}$ and the SOA for the visual stimuli was $85 \mathrm{~ms}$. After the response an isoluminant gray background was presented for $1000 \mathrm{~ms}$. 


\section{Analysis}

Signal detection theory was used to analyze the behavioral results. The rationale behind this is that this way we can verify that the illusions are caused by changes of perceptual sensitivity rather than by the general response bias. This method can describe the sensitivity of the subjects toward the visual stimuli during the process of decision. The sensitivity is expressed as $\mathrm{d}^{\prime}=\mathrm{z}(\mathrm{H})-\mathrm{z}(\mathrm{F})$, where $\mathrm{d}^{\prime}$ is sensitivity, and $\mathrm{z}$ is the inverse cumulative normal. Correct identification of the second flash was recorded as a 'hit' $(\mathrm{H})$; when the subject reported one flash instead of two, it was recorded as a 'miss'. When one flash was reported as two, we accepted it as a 'false alarm' (F) and the correct identification of one flash was accepted as a 'correct rejection'. To calculate the d' value for control we used two subconditions without tones (one flash and two flashes). For fusion we used two sub-conditions with one tone (one flash with one tone and two flashes with one tone) and for double flashes we used two sub-conditions with two tones (one flash with two tones and two flashes with two tones).

To see the power of illusions we compared the control d' value to the $\mathrm{d}^{\prime}$ for fusion or double flash using paired t-test (Watkins et al. 2006) with Bonferroni correction in each condition. Thus we accepted results as significant when the $p<0.025$. Since the strength of the illusions are characterized by this difference, we used these values to test the variance between the conditions with one-way repeated measures ANOVA with Greenhause-Geisser correction in central and peripheral conditions. We used Bonferroni as a post-hoc test.

We calculated a criterion (C) to indicate response bias with the expression

$\mathrm{C}=-[\mathrm{z}(\mathrm{pH})+\mathrm{z}(\mathrm{pF})] / 2($ Macmillan and Creelman 2004)

Thus the positive value of the $\mathrm{C}$ shows the bias when the subjects report rather one, and negative value when two flashes.

Table 1. Criterion and d' values in the condition where stimuli were presented centrally.

\begin{tabular}{|c|c|c|c|c|c|}
\hline \multicolumn{2}{|c|}{ Central condition } & \multirow{2}{*}{$\begin{array}{c}\text { Criterion mean } \\
-0.725\end{array}$} & \multirow{2}{*}{$\begin{array}{l}\text { SEM } \\
0.080\end{array}$} & \multirow{2}{*}{$\begin{array}{c}\text { d' mean } \\
3.376\end{array}$} & \multirow{2}{*}{$\begin{array}{l}\text { SEM } \\
0.344\end{array}$} \\
\hline$H C$ & sensitivity & & & & \\
\hline & fusion & -0.019 & 0.154 & 2.944 & 0.301 \\
\hline & double flash & -1.751 & 0.211 & 1.707 & 0.418 \\
\hline \multirow[t]{3}{*}{$L C$} & sensitivity & -0.442 & 0.148 & 2.904 & 0.290 \\
\hline & fusion & 0.318 & 0.114 & 2.496 & 0.295 \\
\hline & double flash & -1.556 & 0.162 & 1.616 & 0.375 \\
\hline \multirow[t]{3}{*}{ S-iso } & sensitivity & 0.101 & 0.184 & 3.137 & 0.262 \\
\hline & fusion & 0.889 & 0.127 & 2.078 & 0.323 \\
\hline & double flash & -0.947 & 0.226 & 2.139 & 0.326 \\
\hline \multirow[t]{3}{*}{$P$-iso } & sensitivity & -0.324 & 0.134 & 3.325 & 0.346 \\
\hline & fusion & 0.348 & 0.146 & 2.586 & 0.331 \\
\hline & double flash & -1.549 & 0.159 & 2.174 & 0.431 \\
\hline
\end{tabular}

Data are means and standard errors. HC: high contrast, LC: low contrast, S-iso: subjectively isoluminant, P-iso: physically isoluminant

\section{Results}

The detailed data are collected in Table 1, 2, 3 and 4. Here we describe only the relevant statistical results. The criterion showed significant positive bias for fusion and negative bias for double flash compared to control criterion in all condition. This shows that one tone biased the participants to report one flash instead of two for fusion, and two tones biased them to report two instead of one for double flash illusions.

Central presentation: In the high contrast condition, no significant fusion effect was shown, $\mathrm{t}(16)=1.71, \mathrm{p}=0.10$ ), but there was a significant double flash effect after Bonferroni correction, $t(16)=5.06$, $\mathrm{p}<0.001$ (Fig. 3A).

In the low contrast condition, no significant fusion effect was shown, $t(16)=2, p=0.05$, but there was a significant double flash effect, $\mathrm{t}(16)=4.29, \mathrm{p}<0.001$, with the same test (Fig. 3B). In the subjective isoluminant condition, both significant fusion, $\mathrm{t}(16)=5.167, \mathrm{p}<0.001$, and significant double flash effect, $\mathrm{t}(16)=3.72 ; \mathrm{p}<0.01$, were shown (Fig. 3C). 
Table 2. Criterion and $d^{\prime}$ values in the condition where stimuli were presented peripherally.

\begin{tabular}{|c|c|c|c|c|c|}
\hline \multicolumn{2}{|c|}{ Peripheral condition } & \multirow{2}{*}{$\begin{array}{c}\text { Criterion mean } \\
-0.338\end{array}$} & \multirow{2}{*}{$\begin{array}{l}\text { SEM } \\
0.173\end{array}$} & \multirow{2}{*}{$\begin{array}{c}\text { d' mean } \\
3.448\end{array}$} & \multirow{2}{*}{$\begin{array}{l}\text { SEM } \\
0.268\end{array}$} \\
\hline$H C$ & sensitivity & & & & \\
\hline & fusion & 0.613 & 0.213 & 2.602 & 0.353 \\
\hline & double flash & -1.918 & 0.152 & 1.563 & 0.248 \\
\hline \multirow[t]{3}{*}{$L C$} & sensitivity & -0.560 & 0.156 & 2.910 & 0.262 \\
\hline & fusion & 0.482 & 0.157 & 3.169 & 0.400 \\
\hline & double flash & -1.759 & 0.156 & 1.740 & 0.246 \\
\hline \multirow[t]{3}{*}{ S-iso } & sensitivity & -0.176 & 0.187 & 3.118 & 0.322 \\
\hline & fusion & 0.428 & 0.169 & 2.564 & 0.355 \\
\hline & double flash & -1.609 & 0.171 & 1.682 & 0.254 \\
\hline \multirow[t]{3}{*}{$P$-iso } & sensitivity & 0.022 & 0.175 & 2.684 & 0.285 \\
\hline & fusion & 0.776 & 0.163 & 1.994 & 0.275 \\
\hline & double flash & -1.885 & 0.168 & 1.214 & 0.271 \\
\hline
\end{tabular}

Data are means and standard errors. HC: high contrast, LC: low contrast, S-iso: subjectively isoluminant, P-iso: physically isoluminant

Table 3. The results of the statistical comparison concerning the criterion levels under the central condition.

\begin{tabular}{llll}
\hline Central condition & t(16) & p values \\
\hline \multirow{2}{*}{ HC } & fusion & 4.715 & $<0.001$ \\
& double flash & 4.989 & $<0.001$ \\
LC & fusion & 5.178 & $<0.001$ \\
& double flash & 6.673 & $<0.001$ \\
\multirow{2}{*}{-iso } & fusion & 5.492 & $<0.001$ \\
& double flash & 5.311 & $<0.001$ \\
P-iso & fusion & 4.206 & $<0.001$ \\
& double flash & 6.729 & $<0.001$ \\
\hline
\end{tabular}

HC: high contrast, LC: low contrast, S-iso: subjectively isoluminant, $\mathrm{P}$-iso: physically isoluminant

Table 4. The results of the statistical comparison concerning the criterion levels under the pheripheral condition.

\begin{tabular}{llll}
\hline \multicolumn{2}{l}{ Peripheral condition } & t(16) & p values \\
\hline \multirow{2}{*}{ HC } & fusion & 6.084 & $<0.001$ \\
& double flash & 6.250 & $<0.001$ \\
LC & fusion & 4.760 & $<0.001$ \\
& double flash & 7.324 & $<0.001$ \\
S-iso & fusion & 3.584 & $<0.01$ \\
& double flash & 5.618 & $<0.001$ \\
P-iso & fusion & 4.275 & $<0.001$ \\
& double flash & 9.050 & $<0.001$ \\
\hline
\end{tabular}

HC: high contrast, LC: low contrast, S-iso: subjectively isoluminant, $\mathrm{P}$-iso: physically isoluminant
In the physically isoluminant condition, both illusions, the fusion, $\mathrm{t}(16)=2.771, \mathrm{p}<0.05$, and also the double flash, $\mathrm{t}(16)=2.74, \mathrm{p}<0.05$, were significant (Fig. 3D).

The repeated measures ANOVA of the difference scores for the central conditions did not reveal any significant differences between the different conditions (high-contrast, low contrast, subjectively or physically isoluminant), either for the fusion ( $F$ (2.676, $42.81)=1.748, \mathrm{p}=0.17)$ or for double flash ( $\mathrm{F}(2.472$, 39.55)=1.287, $\mathrm{p}=0.29$ ) illusions (Fig. 3E-F).

Peripheral presentation: In the high contrast condition, significant fusion effect, $\mathrm{t}(16)=3.47, \mathrm{p}<0.01$, and double flash effects, $\mathrm{t}(16)=4.86, \mathrm{p}<0.001$, were shown (Fig. 4A).

In the low contrast condition, no significant fusion effect was shown, $\mathrm{t}(16)=0.93, \mathrm{p}=0.36$, but there was a significant double flash effect, $t(16)=3.66, p<0.01$ (Fig. 4B).

In the subjective isoluminant condition, no significant fusion effect was shown, $\mathrm{t}(16)=1.83$, $\mathrm{p}=0.08$, but there was a significant double flash effect, $\mathrm{t}(16)=3.68$, $\mathrm{p}<0.01$ (Fig. 4C).

In the physically isoluminant condition, significant fusion effect, $\mathrm{t}(16)=4.42, \mathrm{p}<0.001$, and also double flash effect, $\mathrm{t}(16)=4.52, \mathrm{p}<0.001$, were shown (Fig. 4D).

The repeated-measures ANOVA of the difference scores for the peripheral conditions showed significant differences between the different conditions (high-contrast, low contrast, subjectively or physically isoluminant) for the fusion effect $(F(2.286,36.58)=$ 
3.898, $\mathrm{p}<0.05$ ), but there were no significant differences between the different conditions for the double flash ( $F(2.684,42.94)=1.653, p=0.19)$ illusion (Fig. 4E-F). In case of the fusion effect the Bonferroni multiple comparison test showed that in the LC condition the difference between the control $\mathrm{d}^{\prime}$ and $\mathrm{d}^{\prime}$ for fusion is bigger than these values in P-iso conditions.

\section{Central presentation}
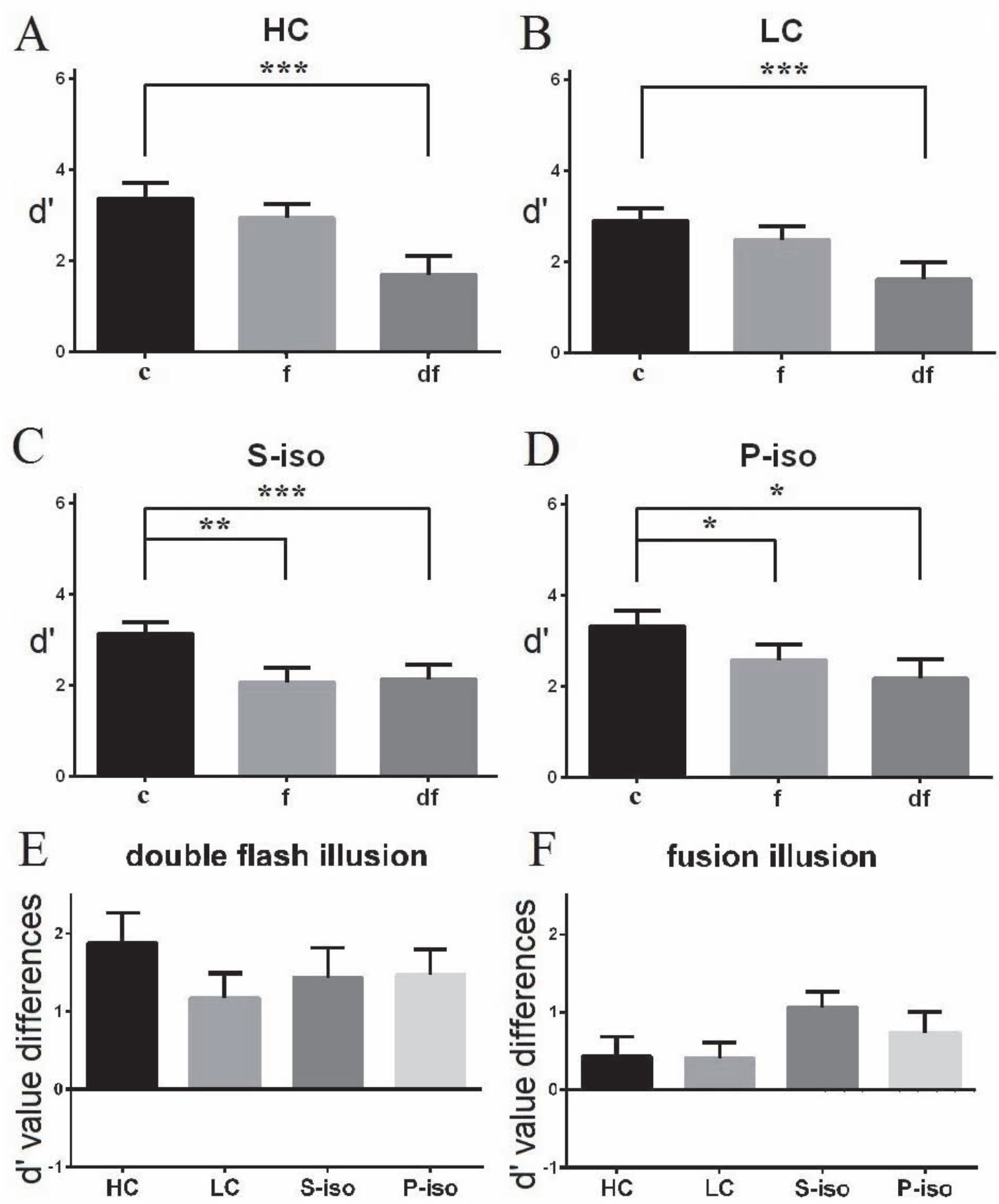

Fig. 3. Results of the psychophysical test in the central condition. The diagram shows the means and standard errors of $d^{\prime}$ 'values and the significant results of the paired t-test in the central conditions. Significant changes $p<0.05(*), p<0.01(* *)$ and $p<0.001(* * *)$ are indicated by asterisks, $n=17$. Panel A: HC: high contrast, panel B: LC: low contrast, panel C: S-iso: subjectively isoluminant, panel D: P-iso: physically isoluminant. Panel $\mathbf{E}$ and $\mathbf{F}$ show the means and standard errors of differences between control and double flash $d^{\prime}$ 'values for double flash (ANOVA, $F(2.472,39.55)=1.287 ; p=0.29 ; n=17$ ) and between control and fusion d' values for fusion (ANOVA, $F(2.676,42.81)=1.748 ; p=0.17 ; n=17)$. c: control, f: fusion, df: double flash 


\section{Peripheral presentation}
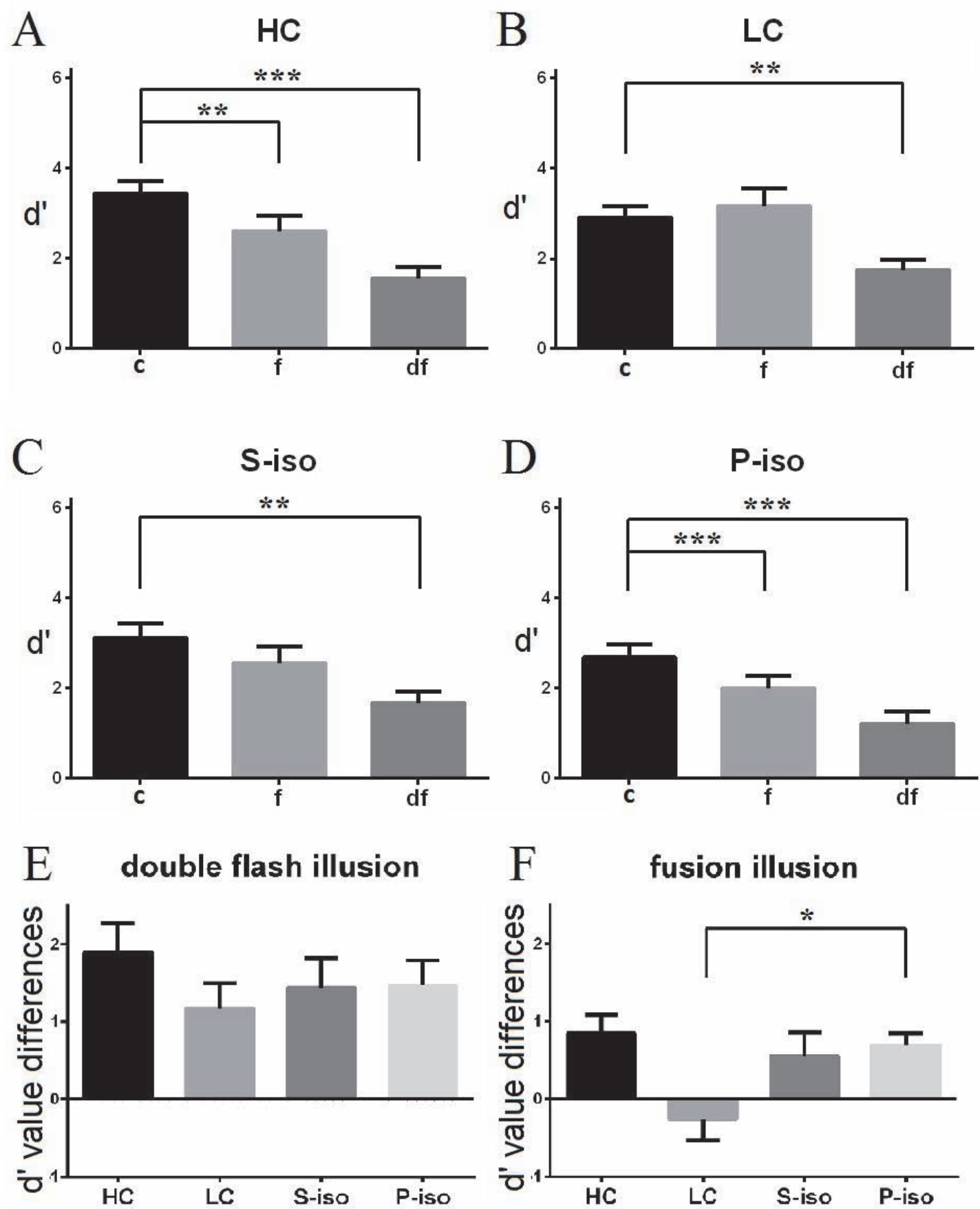

Fig. 4. Results of the psychophysical test in the peripheral condition. The diagram shows the means and standard errors of $d^{\prime}$ values and the significant results of the paired t-test in the peripheral conditions. Significant changes $p<0.05(*), p<0.01(* *)$ and $p<0.001$ $(* * *)$ are indicated by asterisks, $\mathrm{n}=17$. Panel $\mathbf{A}: \mathrm{HC}$ : high contrast, panel $\mathbf{B}$ : LC: low contrast, panel C: $\mathrm{S}$-iso: subjectively isoluminant, panel $\mathbf{D}$ : P-iso: physically isoluminant. Panel $\mathbf{E}$ and $\mathbf{F}$ show the means and standard errors of differences between control and double flash $d^{\prime}$ 'values for double flash (ANOVA, $\left.F(2.684,42.94)=1.653 ; p=0.19 ; n=17\right)$ and between control and fusion d' values for fusion (ANOVA, $F(2.286,36.58)=3.898 ; p<0.05 ; n=17$ ). Bonferroni's multiple comparison test showed that the $L C$ condition is different from P-iso condition for fusion. c: control, f: fusion, df: double flash 


\section{Discussion}

As described earlier the double flash illusion is a very robust phenomenon (Shams et al. 2000). The demonstration of the flash fusion is more difficult because this illusion is fairly weak compared to double flash illusion, the variations in the behavioral performances among participants are quite large; a group of participants did not even report this illusion (Mishra et al. 2008). Thus unless the visibility (modulated by eccentricity and size) of the originally used high contrast disc is extremely poor, the incidence of the flash fusion would be stochastic, depending on the given group of participants (Mishra et al. 2008). Generally, we found the same results as mentioned above with the stimulus set described. The variety of behavioral performance among participants shows a wide range; however, even so we got significant differences for the double flash in all conditions at both central and peripheral stimulus presentations. In some conditions the occurrence of the double flash illusion was more frequent at the peripheral than the central condition, which is consistent with the early results (Bhattacharya et al. 2002).

Previously reported theory suggests that the connection between the primary visual cortex and the STS can play a substantial role in the processing of these illusions. Our aim was to investigate this processing from a different aspect. For this we found driving the different visual pathways a useful approach. We designed stimuli which are matched to the sensitivity of the different pathways. However, we have to note that entirely selective stimulation of the $\mathrm{M}$ or $\mathrm{P}$ pathway is not possible. High contrast stimuli can drive both pathways strongly. Low contrast stimuli can drive the $\mathrm{M}$ pathway separately, but this kind of stimulus is quite weak, so it cannot drive the whole pathway to its full extent. Both the subjective and the physical isoluminant stimuli contain color information, thus they can drive the $\mathrm{P}$ pathway. In addition the subjective isoluminant stimuli are known to be selective for the $\mathrm{P}$ pathway.

To separate the pathways better we used central and peripheral stimulation. The $M$ pathway receives information mainly from the non-central retina through the $\mathrm{M}$ ganglion cells. On the other hand, the $\mathrm{P}$ pathway receives information from the whole retina through the $\mathrm{P}$ ganglion cells, but the density of $\mathrm{P}$ ganglion cells decreases towards the periphery of the retina. Thus, the central stimulation facilitates the processing through $\mathrm{P}$ pathway, while peripheral stimulation drives both pathways. However, our central stimulation cannot stimulate only the $\mathrm{P}$ pathway, because the stimuli, used in other studies and our own as well, are relatively big. There is also a remarkable difference between the retinotopic areas in connecting to other areas, because anatomical connections were found between the primary auditory cortex, superior temporal polysensory area (STP) and the peripheral, retinotopically organized part of the V1 (Clavagnier et al. 2004, Falchier et al. 2002, Rockland and Ojima 2003).

In spite of high variations of the behavioral performance and with the above mentioned restrictions, we found significant differences for the double flash illusion in high contrast conditions with central and peripheral stimulations, which is consistent with previous studies. We also found a strong double flash illusion in the pathway-specific conditions. This indicates that the incongruently added second tone can modulate the visual processing through $\mathrm{M}$ and $\mathrm{P}$ pathways and evokes the illusory perception of a second flash. In case of double flash we did not find dependence on the two pathways, although this could be explained by the robustness of this illusion. The condition, which does not subserve the double flash illusion, might be more sensitive for the differences.

With central stimulation we found a strong significance for fusion in the conditions with red-green color information. These $\mathrm{P}$ pathway optimized (subjectively and physically isoluminant) stimuli are mainly processed through a system having low temporal resolution. This system can be biased easily by the incongruent tone, thus it can fuse the flashes more easily and induce the flash fusion illusion. On the other hand, stimuli optimized for the $M$ pathway are processed through a system having high temporal resolution, which can make distinctions between two flashes easily, thus it cannot sustain the fusion illusion.

With peripheral stimulation we found a strong significance for fusion in the physically isoluminant and in the high contrast conditions. In the high contrast condition the incidence of the flash fusion is not surprising, since it can vary as described earlier, depending on the given group of participants (Mishra et al. 2008). With stimuli optimized for the M pathway we could not induce the fusion illusion. Although we did not find a significant fusion illusion in the subjectively isoluminant condition peripherally, however the difference between the fusion which was found in physical isoluminant condition and the d' level in low 
contrast condition was supported also by the variance analysis.

In conclusion, we found that the robust double flash illusion can be induced on both $\mathrm{M}$ and $\mathrm{P}$ pathways. The fusion illusion can be induced in the $\mathrm{P}$ pathway, while the $\mathrm{M}$ pathway does not support it. Although the difference could be observed only at the peripheral condition, the incidence of flash fusion seems to be pathway-specific depending on the temporal resolution of the given pathway. Thus the origins of the fusion and double flash illusion related activity in STS seem to not identical and it presumes different mechanisms of integration.

\section{Conflict of Interest}

There is no conflict of interest.

\section{Acknowledgements}

This research was supported by the European Union and the State of Hungary, co-financed by the European Social Fund in the framework of TÁMOP 4.2.4. A/2-11-1-20120001 'National Excellence Program' awarded to B.A. and Cs. P. This research was supported by OTKA 83671, TÁMOP-4.2.2/B-10/1-2010-0012. We thank P. Liszli for the technical support and J. Navracsics for proofreading the manuscript.

\section{List of Abbreviations}

Low contrast condition: LC

High contrast condition: HC

Subjectively isoluminant condition: S-iso

Physically isoluminant condition: P-iso

f: fusion

df: double flash

c: control

\section{References}

ANDERSEN TS, TIIPPANA K, SAMS M: Factors influencing audiovisual fission and fusion illusions. Brain Res Cogn Brain Res 21: 301-308, 2004.

BHATTACHARYA J, SHAMS L, SHIMOJO S: Sound-induced illusory flash perception: role of gamma band responses. Neuroreport 13: 1727-1730, 2002.

BILODEAU L, FAUBERT J: Isoluminance and chromatic motion perception throughout the visual field. Vision Res 15: 2073-2081, 1997.

BULLIER J, NOWAK LG: Parallel versus serial processing: new vistas on the distributed organization of the visual system. Curr Opin Neurobio 5: 497-503, 1995.

CLAVAGNIER S, FALCHIER A, KENNEDY H: Long-distance feedback projections to area V1: implications for multisensory integration, spatial awareness, and visual consciousness. Cogn Affect Behav Neurosci 4: 117-126, 2004.

DERRINGTON AM, LENNIE P: Spatial and temporal contrast sensitivities of neurones in lateral geniculate nucleus of macaque. J Physiol 357: 219-240, 1984.

ERNST MO, BANKS MS, BULTHOFF HH: Touch can change visual slant perception. Nat Neurosci 3: 69-73, 2000.

FALCHIER A, CLAVAGNIER S, BARONE P, KENNEDY H: Anatomical evidence of multimodal integration in primate striate cortex. J Neurosci 22: 5749-5759, 2002.

HICKS TP, LEE BB, VIDYASAGAR TR: The responses of cells in macaque lateral geniculate nucleus to sinusoidal gratings. J Physiol 337: 183-200, 1983.

JAEKL PM, SOTO-FARACO S: Audiovisual contrast enhancement is articulated primarily via the M-pathway. Brain Res 1366: 85-92, 2010.

KAPLAN E, SHAPLEY RM: The primate retina contains two types of ganglion cells, with high and low contrast sensitivity. Proc Natl Acad Sci USA 83: 2755-2757, 1986.

KVERAGA K, BOSHYAN J, BAR M: Magnocellular projections as the trigger of top-down facilitation in recognition. J Neurosci 27: 13232-13240, 2007.

LEE BB, WEHRHAHN C, WESTHEIMER G, KREMERS J: The spatial precision of macaque ganglion cell responses in relation to vernier acuity of human observers. Vision Res 35: 2743-2758, 1995.

LEONARDS U, SINGER W: Selective temporal interactions between processing streams with differential sensitivity for colour and luminance contrast. Vision Res 37: 1129-1140, 1997. 
MACMILLAN N, CREELMAN C: Detection Theory: A User's Guide. Psychology Press, Routledge, 2004.

MAUNSELL JH, NEALEY TA, DEPRIEST DD: Magnocellular and parvocellular contributions to responses in the middle temporal visual area MT of the macaque monkey. J Neurosci 10: 3323-3334, 1990.

MEREDITH MA: On the neuronal basis for multisensory convergence: a brief overview. Brain Res Cogn Brain Res 14: 31-40, 2002.

MERIGAN WH, MAUNSELL JH: How parallel are the primate visual pathways? Annu Rev Neurosci 16: 369-402, 1993.

METHA AB, MULLEN KT: Temporal mechanisms underlying flicker detection and identification for red-green and achromatic stimuli. J Opt Soc Am A Opt Image Sci Vis 13: 1969-1980, 1996.

MISHRA J, MARTINEZ A, SEJNOWSKI TJ, HILLYARD SA: Early cross-modal interactions in auditory and visual cortex underlie a sound-induced visual illusion. J Neurosci 27: 4120-4131, 2007.

MISHRA J, MARTINEZ A, HILLYARD SA: Cortical processes underlying sound-induced flash fusion. Brain Res 1242: 102-115, 2008.

ROCKLAND KS, OJIMA H: Multisensory convergence in calcarine visual areas in macaque monkey. Int $J$ Psychophysiol 50: 19-26, 2003.

SHAMS L, KAMITANI Y, SHIMOJO S: Illusions. What you see is what you hear. Nature 408: 788, 2000.

SHAMS L, KAMITANI Y, THOMPSON S, SHIMOJO S: Sound alters visual evoked potentials in humans. Neuroreport 12: 3849-3852, 2001.

SHAMS L, IWAKI S, CHAWLA A, BHATTACHARYA J: Early modulation of visual cortex by sound: an MEG study. Neurosci Lett 378: 76-81, 2005.

SHAPLEY R: Visual sensitivity and parallel retinocortical channels. Annu Rev Psychol 41: 635-658, 1990.

SKOTTUN BC: On using isoluminant stimuli to separate magno- and parvocellular responses in psychophysical experiments - a few words of caution. Behav Res Methods 453: 637-645, 2013.

TOBIMATSU S, TOMODA H, KATO M: Human VEPs to isoluminant chromatic and achromatic sinusoidal gratings: separation of parvocellular components. Brain Topogr 8: 241-243, 1996.

TOOTELL RB, SILVERMAN MS, HAMILTON SL, DE VALOIS RL, SWITKES E: Functional anatomy of macaque striate cortex. III. Color. J Neurosci 8: 1569-1593, 1988.

WATKINS S, SHAMS L, TANAKA S, HAYNES JD, REES G: Sound alters activity in human V1 in association with illusory visual perception. Neuroimage 31: 1247-1256, 2006.

WATKINS S, SHAMS L, JOSEPHS O, REES G: Activity in human V1 follows multisensory perception. Neuroimage 37: 572-578, 2007.

WILSON JT: Interaction of simultaneous visual events. Perception 16: 375-383, 1987.

WOZNY DR, BEIERHOLM UR, SHAMS L: Human trimodal perception follows optimal statistical inference. $J$ Vis 8 : 24. 1-11, 2008. 